\title{
What is the scale of new physics behind the $B$-flavour anomalies?
}

\author{
Luca Di Luzio ${ }^{1, a}$, Marco Nardecchia ${ }^{2, b}$ \\ ${ }^{1}$ Department of Physics, Institute for Particle Physics Phenomenology, Durham University, Durham DH1 3LE, UK \\ 2 Theoretical Physics Department, CERN, Geneva, Switzerland
}

Received: 14 June 2017 / Accepted: 26 July 2017 / Published online: 10 August 2017

(C) The Author(s) 2017. This article is an open access publication

\begin{abstract}
Motivated by the recent hints of lepton flavour non-universality in $B$-meson semi-leptonic decays, we study the constraints of perturbative unitarity on the new physics interpretation of the anomalies in $b \rightarrow c \ell \bar{v}$ and $b \rightarrow s \ell \bar{\ell}$ transitions. Within an effective field theory approach we find that $2 \rightarrow 2$ fermion scattering amplitudes saturate the unitarity bound below 9 and $80 \mathrm{TeV}$, respectively for $b \rightarrow c \ell \bar{v}$ and $b \rightarrow s \ell \bar{\ell}$ transitions. Stronger bounds, up to few $\mathrm{TeV}$, are obtained when the leading effective operators are oriented in the direction of the third generation, as suggested by flavour models. We finally address unitarity constraints on simplified models explaining the anomalies and show that the new physics interpretation is ruled out in a class of perturbative realizations.
\end{abstract}

\section{Contents}

1 Introduction . . . . . . . . . . . . . 1

2 A tale of scales ............... . . 2

3 On the flavour structure of the effective operators . . 4

4 Partial-wave unitarity . . . . . . . . . . 5

5 Unitarity bounds in the EFT . . . . . . . . . 5

6 Unitarity bounds in simplified models . . . . . . . 6

6.1 Colourless vectors . . . . . . . . . . . 7

6.2 Leptoquarks . . . . . . . . . . . . . 8

6.3 Direct searches at the LHC . . . . . . . . 9

7 Conclusions ............... . . 10

A Sample calculation: $S U(2)_{L}$ triplet operator . . . . . 11

References ................ 12

\footnotetext{
a e-mail: luca.di-luzio@durham.ac.uk

b e-mail: marco.nardecchia@cern.ch
}

\section{Introduction}

In the recent years we have witnessed a growing pattern of experimental anomalies in flavour physics, which can be schematically summarized as follows:

1. Semi-leptonic $B$-decays in flavour changing neutral currents (FCNC) $b \rightarrow s \ell \bar{\ell}$, suggesting a deficit of muons compared to electrons. The main observables are: (i) the angular distributions of $B \rightarrow K^{*} \mu \bar{\mu}$ [1-3], (ii) the rate of semi-leptonic decays such as $B \rightarrow K^{*} \mu \bar{\mu}$ [1] and $B_{s} \rightarrow \phi \mu \bar{\mu}[4]$ and (iii) the lepton flavour universality (LFU) violating observables $R_{K}$ [5] and $R_{K^{*}}$ [6], which are defined by the ratios $\mathcal{B}\left(B \rightarrow K^{(*)} \mu \bar{\mu}\right) / \mathcal{B}(B \rightarrow$ $\left.K^{(*)} e \bar{e}\right)$. We remark that the Standard Model (SM) theoretical uncertainty for $R_{K^{(*)}}$ is very small (few percent due to QED radiative corrections [7]). Updated fits based on effective field theory (EFT) analyses, including the most recent $R_{K}{ }^{*}$ measurement, can be found in [8-12].

2. Semi-leptonic $B$-decays in flavour changing charged currents (FCCC) $b \rightarrow c \ell \bar{v}_{\ell}$, suggesting an excess of taus compared to muons and electrons. The main observables are the LFU violating ratios $R_{D^{(*)}}$ [13-15], defined as $\mathcal{B}\left(B \rightarrow D^{(*)} \tau \bar{v}\right) / \mathcal{B}\left(B \rightarrow D^{(*)} \ell \bar{v}\right)$, with $\ell=e, \mu$. In this case it is non-trivial that three different experiments agree well among each other. A recent EFT fit of $R_{D^{(*)}}$ can be found for instance in [16]. Very recently, there has also been a new measurement of $R_{D^{*}}$ by the LHCb collaboration [17], which is remarkably compatible with the previous ones. However, this single measurement does not affect much the global fit.

In both cases the statistical significance reaches the $4 \sigma$ level, while from a theoretical standpoint it is very intriguing that both sets of anomalies can be interpreted within a coherent framework. In particular, one can envisage two orthogonal structures: (i) a vertical (gauge) one: global fits seem to prefer effective operators featuring only $S U(2)_{L}$ doublets and 
(ii) a horizontal (flavour) one: data hints to violation of LFU with a similar hierarchical pattern as in the SM, with new physics contributions negligible in electrons (basically no effects), sizeable in muons (observable only in $b \rightarrow s \mu \bar{\mu}$ ) and large in taus (effects in $b \rightarrow c \tau \bar{\nu}_{\tau}$ and potentially in $b \rightarrow s \nu_{\tau} \bar{\nu}_{\tau}$ ). These facts motivated the community to speculate about the simultaneous explanation of these two sets of anomalies and their connection with the origin of the SM flavour. It is then maybe not too early to dream about new physics and ask "what is the scale of new physics behind the $B$-flavour anomalies?"

Here, we address this question by using an old tool of theoretical physics, namely perturbative unitarity. Perhaps most famously, constraints imposed by perturbative unitarity in $W W$ scattering have been used in the past to infer an upper bound on the Higgs boson mass or, alternatively, on the scale where the SM description of weak interactions needed to be completed in the ultraviolet (UV) in terms of some new strongly coupled dynamics $[18,19]$. What we are going to consider here instead resembles in some sense the Fermi theory of weak interactions [20]. In fact, already in the 1930 s, from the low-energy measurement of $G_{F}$ one could have inferred what was the scale of "new physics" behind the Fermi theory. By looking at $2 \rightarrow 2$ scatterings via fourfermion effective operators in the Fermi theory one finds that unitarity is violated ${ }^{1}$ at energies of the order of $\Lambda_{U}=900$ $\mathrm{GeV}$ (see e.g. [21]). As is well known, the dynamical degrees of freedom of the SM turned out to be weakly coupled and hence much lighter than the unitarity bound, e.g. $M_{W} \ll \Lambda_{U}$.

In this paper, we do something similar to the unitarity analysis in the Fermi theory by considering the four-fermion operators of the $d=6 \mathrm{SM}$-invariant EFT (SMEFT) semi-leptonic basis, under the hypothesis of a short-distance new physics explanation of the experimental anomalies in semi-leptonic $B$-meson decays. ${ }^{2}$ Note that the analysis can be independently carried out for the $b \rightarrow s \ell \bar{\ell}$ and $b \rightarrow c \ell \bar{v}$ anomalies, and we do not necessarily rely on a common explanation of the two. In short, once the Wilson coefficient of an effective operator is fixed by the fit to the anomaly, we can use it in order to extract the scale of unitarity violation without the need of passing through the ambiguous separation of mass vs. coupling. The common lore is that on-shell new degrees of freedom should appear below the scale of unitarity violation (see however [22] for exceptions), with interesting consequences for direct searches at LHC and future colliders.

A simple message that we would like to emphasize is that scattering amplitudes employing SM-invariant effective operators lead to scales of unitarity violation $\Lambda_{U}$ which

\footnotetext{
1 We will sometimes improperly use the term "unitarity violation", by which we mean perturbative unitarity (cf. the discussion in Sect. 4).

${ }^{2}$ Unitarity bounds for the EFT interpretation of $b \rightarrow s \ell \bar{\ell}$ anomalies were briefly mentioned in Ref. [9].
}

are typically smaller than the naive dimensional analysis (NDA) estimate of the strong coupling regime $g_{\star}=4 \pi$, i.e. $M_{\star}=4 \pi \Lambda_{\mathcal{O}}$, where $\Lambda_{\mathcal{O}}$ denotes the scale of the SMinvariant effective operator required to fit the anomaly normalized to unit Wilson coefficient. This enhancement is also in part due to the correlation of the scattering amplitudes in the gauge group space, which is important to take into account when thinking about the energy reach of LHC or future colliders. A related point is the flavour structure of the effective operators. If these are oriented along the third generation fermion families (as motivated in various flavour models), one typically predicts a strong enhancement of the unitarity bound which can even reach few TeV (cf. Table 1).

Similarly to the EFT analysis, unitarity arguments can also be used in order to set perturbativity constraints on the parameter space of simplified models explaining the flavour anomalies. Note, however, that in the latter case the scattering amplitudes do not grow with the energy but reach asymptotic values proportional to the Yukawa-like couplings of the new mediators. It is possible then to translate the unitarity bounds on the coupling into an upper bound on the mass of the new states (once the ratio coupling/mass is fixed in terms of the fit to the relevant anomaly). Remarkably, in some cases the upper bound on the new mediators' mass is so strong that the perturbative interpretation of the anomaly within a given simplified model can be ruled out, or soon tested at the LHC.

The layout of the paper is the following: in Sect. 2 we start by introducing and comparing different kind of scales in the EFT. After discussing in Sect. 3 motivated flavour structures for the effective operators, we briefly introduce the partialwave-unitarity tool in Sect. 4. We continue in Sects. 5 and 6 where we derive the unitarity bounds respectively in the EFT and for simplified models addressing the $B$-flavour anomalies. We finally conclude in Sect. 7, where we also provide a summary of our results. In Appendix A, as a paradigmatic example, we report the details of the unitarity bound calculation in the presence of an $S U(2)_{L}$ triplet effective operator.

\section{A tale of scales}

In what follows we will focus for simplicity on purely lefthanded operators, since they provide the best fit for both anomalies in $b \rightarrow s \mu \bar{\mu}$ and $b \rightarrow c \tau \bar{\nu}$ transitions. The analysis can easily be generalized to scenarios including more operators by using the results given in Sect. 5. In order to start the discussion it is useful to identify and compare four (conceptually different) scales in the EFT: ${ }^{3}$

\footnotetext{
${ }^{3}$ Some of the results presented here will be derived in the following sections.
} 
Table 1 Summary of the different new physics scales associated with the $B$-flavour anomalies in the EFT analysis: $\Lambda_{A}$ is the scale of the effective operator needed to fit the low-energy observable, $\Lambda_{\mathcal{O}}$ is that required by a SMEFT, $\Lambda_{U}$ is the scale of unitarity violation and $M_{\star}$ is the NDA mass scale of the operator in the strongly coupled regime. $\mathcal{O}$ denotes the flavour structure of the triplet operator in Eq. (4), while $\mathrm{FS}_{Q}$ and $\mathrm{FS}_{L}$ are flavour suppression factors in the quark and lepton sector which rescale the aligned entries (those corresponding to $\mathrm{FS}_{Q, L}=1$ ) by a factor $\sqrt{\mathrm{FS}_{Q} \times \mathrm{FS}_{L}}$. The cases marked by $\ddagger$ and $*$ correspond respectively to the ansatz of left-right symmetric partial compositeness and minimal flavour violation in the charged lepton sector (see Sect. 3 for details)

\begin{tabular}{|c|c|c|c|c|c|c|c|}
\hline Anomaly & $\mathcal{O}$ & $\mathrm{FS}_{Q}$ & $\mathrm{FS}_{L}$ & $\Lambda_{A}(\mathrm{TeV})$ & $\left|\Lambda_{\mathcal{O}}\right|(\mathrm{TeV})$ & $\Lambda_{U}(\mathrm{TeV})$ & $M_{\star}(\mathrm{TeV})$ \\
\hline$b \rightarrow c \tau \bar{v}$ & $Q_{23} L_{33}$ & 1 & 1 & 3.4 & 3.4 & 9.2 & 43 \\
\hline$b \rightarrow c \tau \bar{v}$ & $Q_{33} L_{33}$ & $\left|V_{c b}\right|$ & 1 & 3.4 & 0.7 & 1.9 & 8.7 \\
\hline$b \rightarrow s \mu \bar{\mu}$ & $Q_{23} L_{22}$ & 1 & 1 & 31 & 31 & 84 & 390 \\
\hline$b \rightarrow s \mu \bar{\mu}$ & $Q_{33} L_{22}$ & $\left|V_{t s}\right|$ & 1 & 31 & 6.2 & 17 & 78 \\
\hline$b \rightarrow s \mu \bar{\mu}$ & $Q_{33} L_{33}$ & $\left|V_{t s}\right|$ & ${ }^{\ddagger} m_{\mu} / m_{\tau}$ & 31 & 1.5 & 4.1 & 19 \\
\hline$b \rightarrow s \mu \bar{\mu}$ & $Q_{33} L_{33}$ & $\left|V_{t s}\right|$ & $*\left(m_{\mu} / m_{\tau}\right)^{2}$ & 31 & 0.4 & 1.0 & 4.7 \\
\hline
\end{tabular}

1. $\Lambda_{A}$ : the "Fermi constant" of the process.

This is the scale required to explain the anomaly, to be evaluated at the typical energy of the process which is fixed by the $B$-meson mass. The low-energy EFT description is based on $S U(3)_{C} \times U(1)_{E M}$ invariant operators. The index $A$ on $\Lambda_{A}$ runs over the anomalies, schematically $A=\left\{R_{D^{(*)}}, R_{K^{(*)}}\right\}$, and the EFT Lagrangian featuring purely left-handed operators reads

$$
\begin{gathered}
\mathcal{L}_{\text {eff }} \supset-\frac{1}{\Lambda_{R_{D^{(*)}}}^{2}} 2 \bar{c}_{L} \gamma^{\mu} b_{L} \bar{\tau}_{L} \gamma_{\mu} v_{L} \\
+\frac{1}{\Lambda_{R_{K^{*}}}^{2}} \bar{s}_{L} \gamma^{\mu} b_{L} \bar{\mu}_{L} \gamma_{\mu} \mu_{L}+\text { h.c. }
\end{gathered}
$$

where we assumed alignment with the phases of the CKM elements that appear in the corresponding SM operators. Note that the fit of the $R_{D^{(*)}}$ and $R_{K^{(*)}}$ anomalies requires an opposite sign interference with the SM contribution. We also included an extra factor of 2 in the definition of the charged-current operator, so that the latter has the same normalization of the neutral-current operator when considering a SMEFT. The best fit values of the $R_{D^{(*)}}$ [23] and $R_{K^{(*)}}$ [10] anomalies yield respectively

$$
\begin{aligned}
& \Lambda_{R_{D^{(*)}}}=3.4 \pm 0.4 \mathrm{TeV} \\
& \Lambda_{R_{K^{(*)}}}=31 \pm 4 \mathrm{TeV}
\end{aligned}
$$

where the errors are at $1 \sigma$. In the following we will only consider central values.

2. $\Lambda_{\mathcal{O}}$ : the scale of the SMEFT operator.

This is the scale required to explain the anomaly using an EFT at higher energies ${ }^{4}\left(S U(3)_{C} \times S U(2)_{L} \times U(1)_{Y}\right.$ invariant), with Wilson coefficient normalized to one.

\footnotetext{
${ }^{4}$ QCD running effects on the Wilson coefficients are of the order of $1+\frac{\alpha_{s}}{4 \pi} \times \log \frac{\Lambda_{\mathcal{O}}}{m_{b}}$. For $\Lambda_{\mathcal{O}}=1 \mathrm{TeV}$, this corresponds to an $\mathcal{O}(5 \%)$ correction that will be neglected in the following.
}

The index $\mathcal{O}$ on $\Lambda_{\mathcal{O}}$ is associated with an operator of the SMEFT semi-leptonic basis and runs over all the possible Lorentz and flavour structures. For definiteness we will consider here an $S U(2)_{L}$ triplet operator $(Q$ and $L$ denoting $S U(2)_{L}$ doublets)

$$
\begin{aligned}
& \mathcal{L}_{\text {SMEFT }} \supset \frac{1}{\Lambda_{Q_{i j} L_{k l}}^{2}}\left(\bar{Q}_{i} \gamma^{\mu} \sigma^{A} Q_{j}\right)\left(\bar{L}_{k} \gamma_{\mu} \sigma^{A} L_{l}\right) \\
& \quad+\text { h.c., }
\end{aligned}
$$

and two reference flavour structures such that the operator is aligned in the direction of the flavour eigenstates responsible for the anomalies, namely $\mathcal{O}=Q_{23} L_{33}$ (for $b \rightarrow c \tau \bar{\nu}$ transitions) and $\mathcal{O}=Q_{23} L_{22}$ (for $b \rightarrow s \mu \bar{\mu}$ transitions). The matching with Eq. (1) yields

$$
\begin{aligned}
& \left|\Lambda_{Q_{23} L_{33}}\right|=\Lambda_{R_{D^{(*)}}}=3.4 \mathrm{TeV} \\
& \left|\Lambda_{Q_{23} L_{22}}\right|=\Lambda_{R_{K^{(*)}}}=31 \mathrm{TeV}
\end{aligned}
$$

As we will discuss in detail in Sect. 3, depending on the specific flavour ansatz, the scale $\Lambda_{\mathcal{O}}$ can be effectively reduced with respect to the "Fermi constant" of the process. For example, the transition $b \rightarrow c \tau \bar{v}$ could originate from the operator $\mathcal{O}=Q_{33} L_{33}$, where the $3 \rightarrow 2$ transition in the up sector is due to a CKM mixing (in the basis where $Q_{i}=\left(V_{i j}^{\dagger} u_{L}^{j}, d_{L}^{i}\right)^{T}$ ), which yields

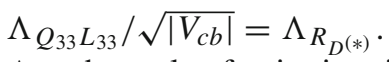

3. $\Lambda_{U}$ : the scale of unitarity violation.

This is the scale where the EFT description breaks down. The important point is that it can be expressed in terms of the scale $\Lambda_{\mathcal{O}}$, without passing through the ambiguous separation between coupling and mass. Using the results of Sect. 5 (which are based on a non-trivial calculation of the scattering amplitude, including gauge group multiplicity factors) we obtain

$$
\Lambda_{U}=\sqrt{\frac{4 \pi}{\sqrt{3}}}\left|\Lambda_{Q_{i j} L_{k l}}\right|,
$$


which yields

$$
\begin{aligned}
& \Lambda_{U}=9.2 \mathrm{TeV} \quad\left(\mathcal{O}=Q_{23} L_{33} \text { case }\right), \\
& \Lambda_{U}=84 \mathrm{TeV} \quad\left(\mathcal{O}=Q_{23} L_{22} \text { case }\right) \text {. }
\end{aligned}
$$

These are the most conservative bounds on the scale of new physics responsible for the anomalies in $b \rightarrow c \tau \bar{v}$ and $b \rightarrow s \mu \bar{\mu}$.

4. $M_{\star}$ : the NDA mass scale in the strongly coupled regime. This is the mass scale associated with the effective operator when saturating perturbativity. After reintroducing $\hbar$ in the NDA (see e.g. [24-26]), one can formally distinguish among scales $(\Lambda)$, masses $(M)$ and couplings $(g)$, and set $M=g \Lambda$. By naively saturating perturbativity at $\left|g_{\star}\right|=4 \pi$, we can write

$$
\frac{1}{\left|\Lambda_{\mathcal{O}}\right|}=\frac{4 \pi}{M_{\star}}
$$

which leads to

$$
\begin{aligned}
& M_{\star}=43 \mathrm{TeV} \quad\left(\mathcal{O}=Q_{23} L_{33} \text { case }\right), \\
& M_{\star}=390 \mathrm{TeV} \quad\left(\mathcal{O}=Q_{23} L_{22} \text { case }\right)
\end{aligned}
$$

Note that $M_{\star}$ is a factor 5 larger than the scale of unitarity violation in Eqs. (8) and (9).

Our results for the EFT analysis are summarized in Table 1 (cf. also Sects. 3-5 for more details of the flavour structure of the effective operators and the unitarity bounds), where we report the values of the four different scales discussed above for the anomalies in either $b \rightarrow c \tau \bar{\nu}$ or $b \rightarrow s \mu \bar{\mu}$ transitions, and depending on the flavour structure of the operator $\mathcal{O}$. The two main points to be observed are the following: (i) $\Lambda_{U}$ is sizeably smaller than $M_{\star}$ and (ii) depending on the flavour structure of the operator $\mathcal{O}$, the scale $\Lambda_{U}$ approaches the energy reach of LHC. This motivates an interesting interplay of the flavour anomalies with direct searches, which is further explored in Sect. 6 by employing simplified models.

\section{On the flavour structure of the effective operators}

The $R_{D^{(*)}}$ and $R_{K^{(*)}}$ anomalies can be interpreted via new physics contributions in quark flavour transitions involving the third and second generation, respectively $b \rightarrow c$ for FCCC and $b \rightarrow s$ for FCNC. In models with motivated flavour structures, it is natural to expect sizeable effects in channels not directly related to the flavour anomalies. In particular, it may happen that operators involving fermions of the third family are enhanced compared to flavour violating ones. This implies that a stronger unitary bound can be derived from $2 \rightarrow 2$ scatterings of fermions of the third generation. For example, when considering the channel related to the anomaly in $b \rightarrow c \tau \bar{\nu}_{\tau}$ we always get a unitarity bound from the scattering $b \bar{c} \rightarrow \tau \bar{\nu}_{\tau}$, but we can reasonably expect that scatterings of the form $b \bar{b} \rightarrow \tau \bar{\tau}$ give stronger unitarity constraints. In order to create a link between the different channels, a flavour structure has to be assumed. In the following, we review some well-known frameworks:

\section{Minimal flavour violation $(M F V)$}

The MFV hypothesis [27] states that the strength of new physics effects are linked to the SM Yukawa couplings, which act as sources of breaking of the enlarged symmetry of the gauge-kinetic terms for fermions, $S U(3)^{3}$ for quarks. In particular, for quark doublets we see that flavour violating interactions are generated at the leading order (in powers of Yukawas) by

$\bar{Q}_{i}\left(a Y_{U} Y_{U}^{\dagger}+b Y_{D} Y_{D}^{\dagger}\right)_{i j} Q_{j}$

where we omitted $S U(2)_{L}$ and Lorentz indices. Here, $a$ and $b$ are coefficients of similar size. This implies a suppression of flavour violating quark currents compared to flavour conserving ones

$\frac{\bar{c}_{L} \gamma^{\mu} b_{L}}{\bar{t}_{L} \gamma^{\mu} b_{L}} \sim \frac{V_{c b}}{V_{t b}} \simeq V_{c b}, \quad \frac{\bar{s}_{L} \gamma^{\mu} b_{L}}{\bar{b}_{L} \gamma^{\mu} b_{L}} \sim \frac{V_{t s}^{*}}{V_{t b}^{*}} \simeq V_{t s}^{*}$.

2. $S U(2)_{Q}$ flavour symmetry

In the limit of vanishing SM Yukawas for the first two quark generations, an $S U(2)^{3}$ global symmetry is restored. This approximate symmetry (or a subgroup of it) might be promoted to be a fundamental symmetry in the UV. In particular, there might be an $S U(2)_{Q}$ symmetry that distinguishes the quark doublets of the first two generations from the third one, and which has to be eventually broken in order to reproduce the observed pattern of SM masses and mixings. If the breaking is achieved via a spurion field $\vec{X}$ that transforms as the fundamental representation of $S U(2)_{Q}$, we get (see e.g. [28]) that the the typical size of $|\vec{X}|$ is of $\mathcal{O}\left(\lambda^{2}\right)$, where $\lambda \sim 0.2$ is the Cabibbo angle. In this case, we also expect that new physics effects in FCCC and FCNC scale like

$\frac{\bar{c}_{L} \gamma^{\mu} b_{L}}{\bar{t}_{L} \gamma^{\mu} b_{L}} \sim \lambda^{2}, \quad \frac{\bar{s}_{L} \gamma^{\mu} b_{L}}{\bar{b}_{L} \gamma^{\mu} b_{L}} \sim \lambda^{2}$.

3. Partial compositeness (PC)

A dynamical explanation of the flavour structure of the SM is provided by the paradigm of PC [29] in the context of composite Higgs models. In this framework the SM fields are linear combinations of elementary and composite states. The admixture elementary-composite of every 
SM state is regulated by a parameter $\epsilon_{i}^{A}$, where $A$ runs over the SM fermion fields $(A=Q, L, u, d, e)$ and $i$ is a family index. In terms of the mixing parameters, the Yukawas of the SM are given by $\left(Y_{U}\right)_{i j} \sim \epsilon_{i}^{Q} \epsilon_{j}^{u}$ and $\left(Y_{D}\right)_{i j} \sim \epsilon_{i}^{Q} \epsilon_{j}^{d}$. It is possible to show (see e.g. [30]) that the $\epsilon_{i}^{Q}$ are linked to the size of the CKM matrix elements, i.e. $\epsilon_{2} / \epsilon_{3} \sim \lambda^{2}$ and $\epsilon_{1} / \epsilon_{3} \sim \lambda^{3}$. New physics effects are hence related to the size of the $\epsilon_{i}^{A}$ coefficients, and for quark left-handed currents one expects a similar scaling for FCCC and FCNC as in Eq. (15).

We conclude that for all the three frameworks above the transition between the third and second generation is suppressed by a factor $\mathcal{O}\left(\lambda^{2}\right)$ compared to the diagonal case involving only the third family. This implies that stronger unitarity bound can be derived from $2 \rightarrow 2$ scattering of the third family. For the presentation of our results in Table 1 we fix the numerical values to the MFV case, leading to a $\left|V_{c b}\right|$ suppression in FCCC and a $\left|V_{t s}\right|$ one in FCNC.

On the other hand, the situation in the lepton sector crucially depends on the unknown origin of neutrino masses. Note that the new physics effects required by the $B$-flavour anomalies do not violate the accidental $U(1)_{e, \mu, \tau}$ symmetry of the SM which arises in the $m_{v} \rightarrow 0$ limit (or, equivalently, in the decoupling limit of lepton-number-violating effective operators). It is hence reasonable to assume that the source of LFU breaking required by the $B$-flavour anomalies is connected to the charged lepton masses. Two structures can easily be motivated:

$$
\begin{aligned}
& \frac{\bar{\ell}_{L}^{i} \gamma^{\mu} \ell_{L}^{i}}{\bar{\ell}_{L}^{j} \gamma^{\mu} \ell_{L}^{j}} \sim \frac{\left(\epsilon_{i}^{L}\right)^{2}}{\left(\epsilon_{j}^{L}\right)^{2}} \sim \frac{m_{\ell_{i}}}{m_{\ell_{j}}} \text { or } \\
& \frac{\bar{\ell}_{L}^{i} \gamma^{\mu} \ell_{L}^{i}}{\bar{\ell}_{L}^{j} \gamma^{\mu} \ell_{L}^{j}} \sim \frac{\left(Y_{E} Y_{E}^{\dagger}\right)_{i i}}{\left(Y_{E} Y_{E}^{\dagger}\right)_{j j}} \sim\left(\frac{m_{\ell_{i}}}{m_{\ell_{j}}}\right)^{2},
\end{aligned}
$$

where the first option corresponds to PC with $\epsilon_{i}^{L} \sim \epsilon_{i}^{e}$ (implying $\left(Y_{E}\right)_{i j} \sim \epsilon_{i}^{L} \epsilon_{j}^{L}$ ) and the second one to MFV in the charged lepton sector. In Table 1 we use these two benchmarks, though different patterns can be of course envisaged.

\section{Partial-wave unitarity}

Here we briefly recap the partial-wave unitarity formalism. More details can be found e.g. in [31,32]. Let us denote by $\mathcal{M}_{f i}(\sqrt{s}, \cos \theta)$ the matrix element of a $2 \rightarrow 2$ scattering amplitude in momentum space, where $\sqrt{s}$ is the center of mass energy and $\theta$ is the azimuthal angle of the scattering. The dependence from $\cos \theta$ can be eliminated by projecting the amplitude onto partial waves of total angular momentum $J$. In our case it suffices to consider the lowest partial wave, defined by $a_{f i}^{0}=\frac{1}{32 \pi s} \int_{-1}^{1} \mathrm{~d}(\cos \theta) \mathcal{M}_{f i}(\sqrt{s}, \cos \theta)$.

This expression is only valid in the high-energy limit, since we neglected kinematical factors ensuring that the partial wave is zero at threshold (see e.g. [33]). The right hand side of Eq. (17) must be further multiplied by a $\frac{1}{\sqrt{2}}$ factor for any identical pair of particles either in the initial or final state. The unitarity of the $S$-matrix implies

$\frac{1}{2 i}\left(a_{f i}^{0}-a_{i f}^{0 *}\right) \geq \sum_{h} a_{h f}^{0 *} a_{h i}^{0}$,

where the inequality originates from the fact that we restricted the sum over $h$ to 2-particle states. For $i=f$ Eq. (18) reduces to $\operatorname{Im} a_{i i}^{0} \geq\left|a_{i i}^{0}\right|^{2}$ or, equivalently, $\left|\operatorname{Im} a_{i i}^{0}\right| \leq 1$ and $\left|\operatorname{Re} a_{i i}^{0}\right| \leq \frac{1}{2}$. It is customary to define the perturbative unitarity bound

$\left|\operatorname{Re}\left(a_{i i}^{0}\right)^{\text {Born }}\right| \leq \frac{1}{2}$,

at the level of the Born amplitude. Although the choice in Eq. (19) is somewhat arbitrary, it yields a reasonable indication of the range of validity of the perturbative expansion. In fact, a Born value of $\operatorname{Re} a_{i i}^{0}=\frac{1}{2}$ and $\operatorname{Im} a_{i i}^{0}=0$ needs at least a higher-order correction of $40 \%$ in order to restore unitarity (see e.g. [33]), thus signalling the breakdown of the expansion itself.

It is also useful to note that in order to optimize the unitarity bound one can look for correlations in the partial-wave matrix (e.g. in the gauge group or flavour space). This corresponds to diagonalizing the partial-wave matrix and setting the bound on the largest eigenvalue, $\operatorname{Re} \tilde{a}_{i i}^{0}<1 / 2$, with the forward scattering $i=f$ understood to correspond to a superposition of states which is an eigenvector of $\tilde{a}_{i i}^{0}$. Note that neglecting a scattering channel for the partial-wave matrix corresponds to removing the associated row/column. Thanks to the Cauchy interlacing theorem, we also know that the largest eigenvalue of the reduced matrix is always $\leq$ than the largest eigenvalue of the full matrix. Hence, by neglecting a scattering channel the unitarity bound still (conservatively) applies.

\section{Unitarity bounds in the EFT}

In this section we derive the connection between the scale of unitarity violation $\Lambda_{U}$ and the coefficients $\Lambda_{\mathcal{O}}$ of the semi-leptonic SMEFT basis, relevant for the $R_{D^{(*)}}$ and $R_{K^{(*)}}$ anomalies. At energies $\sqrt{s} \gg v$ the scattering amplitudes are conveniently described by exploiting the full SM invariance. A complete basis of semi-leptonic $d=6$ operators invariant under the SM gauge symmetry is [23] 
Table 2 Scale of unitarity violation $\Lambda_{U}$ as a function of the coefficients $\Lambda_{\mathcal{O}}$ of the semi-leptonic SMEFT basis of Eq. (20). For the case of $Q_{L} \bar{Q}_{L} \rightarrow L_{L} \bar{L}_{L}$ scattering the $S U(2)_{L}$ triplet and singlet channels are labelled explicitly. The third column denotes the enhancement factors on the partial wave due to the gauge group structure in

$S U(3)_{C} \times S U(2)_{L}$ space

\begin{tabular}{lll}
\hline Scattering & $\Lambda_{U}$ & $S U(3)_{C} \times S U(2)_{L}$ \\
\hline$\left(Q_{L}+\bar{Q}_{L}\right)_{3} \rightarrow\left(L_{L}+\bar{L}_{L}\right)_{3}$ & $\sqrt{\frac{4 \pi}{\sqrt{3}}}\left|\Lambda_{Q L^{(3)}}\right|$ & $\sqrt{3} \times 2$ \\
$\left(Q_{L}+\bar{Q}_{L}\right)_{1} \rightarrow\left(L_{L}+\bar{L}_{L}\right)_{1}$ & $\sqrt{\frac{4 \pi}{\sqrt{3}}}\left|\Lambda_{Q L^{(1)}}\right|$ & $\sqrt{3} \times 2$ \\
$u_{R}+\bar{u}_{R} \rightarrow e_{R}+\bar{e}_{R}$ & $\sqrt{\frac{8 \pi}{\sqrt{3}}}\left|\Lambda_{u e}\right|$ & $\sqrt{3}$ \\
$d_{R}+\bar{d}_{R} \rightarrow e_{R}+\bar{e}_{R}$ & $\sqrt{\frac{8 \pi}{\sqrt{3}}}\left|\Lambda_{d e}\right|$ & $\sqrt{3}$ \\
$u_{R}+\bar{u}_{R} \rightarrow L_{L}+\bar{L}_{L}$ & $\sqrt{\frac{8 \pi}{\sqrt{6}}}\left|\Lambda_{u L}\right|$ & $\sqrt{3} \times \sqrt{2}$ \\
$d_{R}+\bar{d}_{R} \rightarrow L_{L}+\bar{L}_{L}$ & $\sqrt{\frac{8 \pi}{\sqrt{6}}}\left|\Lambda_{d L}\right|$ & $\sqrt{3} \times \sqrt{2}$ \\
$Q_{L}+\bar{Q}_{L} \rightarrow e_{R}+\bar{e}_{R}$ & $\sqrt{\frac{8 \pi}{\sqrt{6}}}\left|\Lambda_{Q e}\right|$ & $\sqrt{3} \times \sqrt{2}$ \\
$d_{R}+\bar{Q}_{L} \rightarrow L_{L}+\bar{e}_{R}$ & $\sqrt{\frac{8 \pi}{\sqrt{3}}}\left|\Lambda_{d Q L e}\right|$ & $\sqrt{3}$ \\
$Q_{L}+\bar{u}_{R} \rightarrow L_{L}+\bar{e}_{R}$ & $\sqrt{\frac{8 \pi}{\sqrt{3}}}\left|\Lambda_{Q u L e}\right|$ & $\sqrt{3}$ \\
\hline
\end{tabular}

$$
\begin{aligned}
\mathcal{L}_{\text {SMEFT }} \supset & \frac{1}{\Lambda_{Q L^{(3)}}^{2}}\left(\bar{Q}_{L} \gamma_{\mu} \sigma^{A} Q_{L}\right)\left(\bar{L}_{L} \gamma^{\mu} \sigma^{A} L_{L}\right) \\
& +\frac{1}{\Lambda_{Q L^{(1)}}^{2}}\left(\bar{Q}_{L} \gamma_{\mu} Q_{L}\right)\left(\bar{L}_{L} \gamma^{\mu} L_{L}\right) \\
& +\frac{1}{\Lambda_{u e}^{2}}\left(\bar{u}_{R} \gamma_{\mu} u_{R}\right)\left(\bar{e}_{R} \gamma^{\mu} e_{R}\right) \\
& +\frac{1}{\Lambda_{d e}^{2}}\left(\bar{d}_{R} \gamma_{\mu} d_{R}\right)\left(\bar{e}_{R} \gamma^{\mu} e_{R}\right) \\
& +\frac{1}{\Lambda_{u L}^{2}}\left(\bar{u}_{R} \gamma_{\mu} u_{R}\right)\left(\bar{L}_{L} \gamma^{\mu} L_{L}\right) \\
& +\frac{1}{\Lambda_{d L}^{2}}\left(\bar{d}_{R} \gamma_{\mu} d_{R}\right)\left(\bar{L}_{L} \gamma^{\mu} L_{L}\right) \\
& +\frac{1}{\Lambda_{Q e}^{2}}\left(\bar{Q}_{L} \gamma_{\mu} Q_{L}\right)\left(\bar{e}_{R} \gamma^{\mu} e_{R}\right) \\
& +\frac{1}{\Lambda_{d Q L e}^{2}}\left(\bar{d}_{R} Q_{L}\right)\left(\bar{L}_{L} e_{R}\right) \\
& +\frac{1}{\Lambda_{Q u L e}^{2}}\left(\bar{Q}_{L} u_{R}\right) i \sigma^{2}\left(\bar{L}_{L} e_{R}\right) \\
& +\frac{1}{\Lambda_{Q u L e^{\prime}}^{2}}\left(\bar{Q}_{L} \sigma_{\mu \nu} u_{R}\right) i \sigma^{2}\left(\bar{L}_{L} \sigma_{\mu \nu} e_{R}\right)+\text { h.c. },
\end{aligned}
$$

where flavour indices have been suppressed. Here, $Q_{L}$ and $L_{L}$ denote $S U(2)_{L}$ doublets, while $u_{R}, d_{R}$ and $e_{R}$ are $S U(2)_{L}$ singlets.

An important aspect to be taken into account for the determination of the unitarity bound is the correlation of the scattering amplitude in the $S U(3)_{C} \times S U(2)_{L}$ space. Let us consider, for instance, the scattering $\left(Q_{L}\right)_{a}^{\alpha}+\left(\bar{Q}_{L}\right)_{b}^{\beta} \rightarrow$ $\left(L_{L}\right)_{c}+\left(\bar{L}_{L}\right)_{d}$, where greek (latin) indices run over the fundamental of $S U(3)_{C}\left(S U(2)_{L}\right)$. Assuming a colour singlet channel (which applies to all the operators in Eq. (20)) the amplitude in colour space can be represented by a $4 \times 4$ matrix in the basis $\left\{\left(Q_{L}\right)^{1}\left(\bar{Q}_{L}\right)^{1},\left(Q_{L}\right)^{2}\left(\bar{Q}_{L}\right)^{2},\left(Q_{L}\right)^{3}\left(\bar{Q}_{L}\right)^{3},\left(L_{L}\right)\right.$ $\left.\left(\bar{L}_{L}\right)\right\}$. Similarly, in $S U(2)_{L}$ space we can represent it via a $4 \times 4$ matrix in the basis $\left\{\psi_{1} \bar{\psi}_{1}, \psi_{1} \bar{\psi}_{2}, \psi_{2} \bar{\psi}_{1}, \psi_{2} \bar{\psi}_{2}\right\}$, where $\psi_{a}\left(a=1,2\right.$ being an $S U(2)_{L}$ index) denotes either $\left(Q_{L}\right)^{\alpha}$ or $L_{L}$. A stronger unitarity bound can be hence obtained by preparing the initial and final states of the scattering in the eigenstate corresponding to the highest eigenvalue of $a^{0}$ both in $S U(3)_{C}$ and $S U(2)_{L}$ space (cf. also the discussion at the end of Sect. 4). By looking at the different scattering channels displayed in the first column of Table 2, we obtain for each case the scale of unitarity violation $\Lambda_{U}$ (defined as the value of $\sqrt{s}$ where the condition in Eq. (19) is saturated) as a function of the scale of the SMEFT operator $\Lambda_{\mathcal{O}}$, where $\mathcal{O}=\left\{Q L^{(3)}, Q L^{(1)}, \ldots\right\}$. In the last column of Table 2 we also show the enhancement of the $a^{0}$ eigenvalue due to the $S U(3)_{C} \times S U(2)_{L}$ group structure of the partial wave. The full calculation of the unitarity bound for the triplet operator $\mathcal{O}=Q L^{(3)}$ (including a detailed discussion of the gauge group enhancement) is exemplified in Appendix A, while the bounds for the other cases are obtained in a similar way. We finally observe that since the tensor operator does not contribute to the $J=0$ partial wave, in order to apply the unitarity bound from $\Lambda_{Q u L e^{\prime}}$ one would need to inspect higher partial waves.

\section{Unitarity bounds in simplified models}

We continue by applying unitarity constraints on the parameter space of simplified models for the explanation of the $R_{D^{(*)}}$ and $R_{K^{(*)}}$ anomalies. Note that this case is slightly different from the unitarity bounds in the EFT, since the scattering amplitudes do not grow with the energy. Still, one can examine the $2 \rightarrow 2$ scatterings of SM fermions in order to set perturbativity limits on the renormalizable couplings of the new 
Table 3 Overview of simplified models which can possibly contribute to $R_{D^{(*)}}$ or $R_{K^{(*)}}$ via a singlet/triplet left-handed operator. Only for specific values of the ratio of the Wilson coefficients $c_{1} / c_{3}$ (obtained by integrating out a given mediator) the dangerous $d_{i} \rightarrow d_{j} v \bar{v}$ operators are not generated ( $U_{1}$ case)

\begin{tabular}{lllllll}
\hline Simplified model & Spin & SM irrep & $c_{1} / c_{3}$ & $R_{D^{(*)}}$ & $R_{K^{(*)}}$ & No $d_{i} \rightarrow d_{j} \nu \bar{v}$ \\
\hline$Z^{\prime}$ & 1 & $(1,1,0)$ & $\infty$ & $\times$ & $\checkmark$ & $\times$ \\
$V^{\prime}$ & 1 & $(1,3,0)$ & 0 & $\checkmark$ & $\checkmark$ & $\times$ \\
$S_{1}$ & 0 & $(\overline{3}, 1,1 / 3)$ & -1 & $\checkmark$ & $\checkmark$ & $\times$ \\
$S_{3}$ & 0 & $(\overline{3}, 3,1 / 3)$ & 3 & $\checkmark$ & $\checkmark$ & $\checkmark$ \\
$U_{1}$ & 1 & $(3,1,2 / 3)$ & 1 & $\checkmark$ & $\checkmark$ \\
$U_{3}$ & 1 & $(3,3,2 / 3)$ & -3 & & $\times$ \\
\hline
\end{tabular}

mediators and, in turn, translate them into an upper bound on the mass of the new states (once the ratio coupling/mass is fixed in terms of the fit to the relevant observable). As two representative classes of simplified models, we consider colourless spin-1 mediators and scalar/vector leptoquarks.

However, some comments are in order about the phenomenological viability of the simplified models. The criterium that we are going to follow in order to select the suitable representations for the new mediators is that after integrating them out they are able to generate triplet and singlet left-handed operator, namely those associated with the coefficients $\Lambda_{Q L^{(3)}}$ and $\Lambda_{Q L^{(1)}}$ in Eq. (20). In all the cases that we are going to consider the phenomenologically disfavoured right-handed and scalar/tensor operator of Eq. (20) can be set to zero by a proper choice of the mediator's coupling. Given these conditions, the full set of simplified models is displayed in Table 3 .

From the $S U(2)_{L}$ decomposition (neglecting flavour indices and reinserting the Wilson coefficients explicitly)

$$
\begin{aligned}
& \frac{c_{1}}{\Lambda^{2}}\left(\bar{Q}_{L} \gamma_{\mu} Q_{L}\right)\left(\bar{L}_{L} \gamma^{\mu} L_{L}\right)+\frac{c_{3}}{\Lambda^{2}}\left(\bar{Q}_{L} \gamma_{\mu} \sigma^{A} Q_{L}\right)\left(\bar{L}_{L} \gamma^{\mu} \sigma^{A} L_{L}\right) \\
& =\frac{c_{1}+c_{3}}{\Lambda^{2}}\left[\left(\bar{d}_{L} \gamma_{\mu} d_{L}\right)\left(\bar{e}_{L} \gamma^{\mu} e_{L}\right)+\left(\bar{u}_{L} \gamma_{\mu} u_{L}\right)\left(\bar{v}_{L} \gamma^{\mu} v_{L}\right)\right] \\
& \quad+\frac{c_{1}-c_{3}}{\Lambda^{2}}\left[\left(\bar{d}_{L} \gamma_{\mu} d_{L}\right)\left(\bar{v}_{L} \gamma^{\mu} v_{L}\right)+\left(\bar{u}_{L} \gamma_{\mu} u_{L}\right)\left(\bar{e}_{L} \gamma^{\mu} e_{L}\right)\right] \\
& \quad+2 \frac{c_{3}}{\Lambda^{2}}\left[\left(\bar{u}_{L} \gamma_{\mu} d_{L}\right)\left(\bar{e}_{L} \gamma^{\mu} v_{L}\right)+\left(\bar{d}_{L} \gamma_{\mu} u_{L}\right)\left(\bar{v}_{L} \gamma^{\mu} e_{L}\right)\right]
\end{aligned}
$$

it is evident that for $c_{1} / c_{3}=-1$ there are no $b \rightarrow s \mu \bar{\mu}$ transitions. Similarly, for $c_{1} / c_{3}=1$ processes of the type $d_{i} \rightarrow$ $d_{j} \nu \bar{\nu}$ are absent. The latter are particularly dangerous, since decays like $B \rightarrow K^{(*)} \nu \bar{v}$ or $K \rightarrow \pi \nu \bar{\nu}$ are very constraining $[34,35]$. From this point of view $U_{1}$ is phenomenologically favoured, since it automatically ensures the absence of $d_{i} \rightarrow$ $d_{j} v \bar{v}$ operators at the scale of the threshold. ${ }^{5}$ For an incomplete list of references addressing both $R_{D^{(*)}}$ and $R_{K^{(*)}}$ with this leptoquark see [37-39]. Other phenomenological issues

\footnotetext{
5 This can also be achieved in non-minimal scenarios with two leptoquarks via a proper cancellation [36].
}

that have to be taken into account when considering a simplified model are electroweak precision tests and the radiative generation of LFU breaking effects in $Z$ and $\tau$ decays $[40,41]$. In order to avoid those bounds one has to assume either a certain level of tuning within the couplings of the simplified model or rely on some non-generic features of the UV completion of the simplified model. For an example of a leptoquark model where all these bounds have been consistently addressed see e.g. [42]. Finally, one has to consider direct searches that we briefly address in Sect. 6.3. Our results on the unitarity bounds for colourless vectors and leptoquarks, which are summarized in Tables 4 and 5, provide an extra constraint which has to be satisfied within perturbative models.

\subsection{Colourless vectors}

Let us first consider the case of a real electroweak vector, $V_{\mu}^{\prime} \sim(1,3,0)$, which couples to the SM fermions via

$\mathcal{L}_{V^{\prime}} \supset \lambda_{i j}^{Q} \bar{Q}_{i} \gamma^{\mu} \sigma^{A} Q_{j} V_{\mu}^{\prime A}+\lambda_{i j}^{L} \bar{L}_{i} \gamma^{\mu} \sigma^{A} L_{j} V_{\mu}^{\prime A}+$ h.c.

At energies $\sqrt{s} \gg M_{V^{\prime}}$ the partial-wave scattering matrix in the $\left(Q_{j} \bar{Q}_{i}, L_{l} \bar{L}_{k}\right)$ basis is given by ${ }^{6}$

$a^{0}=\frac{1}{8 \pi}\left(\begin{array}{cc}3\left|\lambda_{i j}^{Q}\right|^{2} & \sqrt{3} \lambda_{i j}^{Q}\left(\lambda_{k l}^{L}\right)^{*} \\ \sqrt{3}\left(\lambda_{i j}^{Q}\right)^{*} \lambda_{k l}^{L} & \left|\lambda_{k l}^{L}\right|^{2}\end{array}\right)$,

where we also took into account the $S U(3)_{C} \times S U(2)_{L}$ multiplicity factors. The formalism for extracting the correlation in the gauge group space follows very closely the sample calculation of the scattering with the effective triplet operator, which is detailed in Appendix A. The largest eigenvalue of Eq. (23) is

\footnotetext{
${ }^{6}$ An extra channel with $V^{\prime} V^{\prime}$ in the initial/final state opens up at energies $\sqrt{s}>2 M_{V^{\prime}}$. By neglecting such contribution, the unitarity bound obtained by considering the reduced partial-wave matrix conservatively applies (cf. the discussion at the end of Sect. 4).
} 
Table 4 Summary of unitarity bounds for colourless spin-1 mediators. $\mathrm{FS}_{Q}$ and $\mathrm{FS}_{L}$ denote the flavour suppression factors in the quark and lepton sectors (same as in Table 1)

\begin{tabular}{llllll}
\hline Anomaly & Coupling & $\mathrm{FS}_{Q}$ & $\mathrm{FS}_{L}$ & $M_{V^{\prime}}(\mathrm{TeV})$ & $M_{Z^{\prime}}(\mathrm{TeV})$ \\
\hline$b \rightarrow c \tau \bar{\nu}$ & $\lambda_{23}^{Q} \lambda_{33}^{L}$ & 1 & 1 & 6.5 & $\times$ \\
$b \rightarrow c \tau \bar{\nu}$ & $\lambda_{33}^{Q} \lambda_{33}^{L}$ & $\left|V_{c b}\right|$ & 1 & 1.3 & $\times$ \\
$b \rightarrow s \mu \bar{\mu}$ & $\lambda_{23}^{Q} \lambda_{22}^{L}$ & 1 & 1 & 59 & 59 \\
$b \rightarrow s \mu \bar{\mu}$ & $\lambda_{33}^{Q} \lambda_{22}^{L}$ & $\left|V_{t s}\right|$ & 1 & 12 & 12 \\
$b \rightarrow s \mu \bar{\mu}$ & $\lambda_{33}^{Q} \lambda_{33}^{L}$ & $\left|V_{t s}\right|$ & $m_{\mu} / m_{\tau}$ & 2.9 & 2.9 \\
$b \rightarrow s \mu \bar{\mu}$ & $\lambda_{33}^{Q} \lambda_{33}^{L}$ & $\left|V_{t s}\right|$ & $\left(m_{\mu} / m_{\tau}\right)^{2}$ & 0.7 & 0.7 \\
\hline
\end{tabular}

$a^{0}=\frac{3\left|\lambda_{i j}^{Q}\right|^{2}+\left|\lambda_{k l}^{L}\right|^{2}}{8 \pi}$,

and the associated unitarity bound reads

$3\left|\lambda_{i j}^{Q}\right|^{2}+\left|\lambda_{k l}^{L}\right|^{2}<4 \pi$

Note that this is stronger than the perturbativity bound sometimes quoted in the literature, e.g. $\left|\lambda_{i j}^{Q, L}\right|<\sqrt{4 \pi}$ [43]. In the following, we exemplify the unitarity bounds in the case where the couplings of $V^{\prime}$ are aligned with the operators responsible for $R_{D^{(*)}}$ and $R_{K^{(*)}}$, respectively $\lambda_{23}^{Q} \lambda_{33}^{L}$ and $\lambda_{23}^{Q} \lambda_{22}^{L}$. This actually yields the most conservative bounds without flavour enhancements. The generalization to nonaligned cases is straightforward and it is reported in Table 4 for some representative cases. In this respect, we note that the multiple coupling configuration with $\lambda_{33}^{Q} \sim \lambda_{23}^{Q}$ might help in relaxing the bounds from Refs. [40,41]. Integrating out the $V^{\prime}$ and matching with Eq. (1), we obtain

$\frac{\lambda_{23}^{Q} \lambda_{33}^{L}}{M_{V^{\prime}}^{2}}=\frac{1}{\Lambda_{R_{D^{(*)}}}^{2}}, \quad-\frac{\lambda_{23}^{Q} \lambda_{22}^{L}}{M_{V^{\prime}}^{2}}=\frac{1}{\Lambda_{R_{K^{(*)}}}^{2}}$.

It is convenient to define the auxiliary functions

$r=\left|\frac{\lambda_{k l}^{L}}{\lambda_{i j}^{Q}}\right|$ and $f(r)=\frac{r}{3+r^{2}}$,

so that the bound in Eq. (25) can be recast as (using also Eq. (26))

$M_{V^{\prime}}<\sqrt{4 \pi f(r)} \Lambda_{A}$,

where $A=\left\{R_{D^{(*)}}, R_{K^{(*)}}\right\}$. The most conservative bound is obtained by maximizing the function $f(r)$ at $r=\sqrt{3}$, which yields

$M_{V^{\prime}}<\sqrt{\frac{2 \pi}{\sqrt{3}}} \Lambda_{A}=6.5 \mathrm{TeV}(59 \mathrm{TeV})$

for the case of $R_{D^{(*)}}\left(R_{K^{(*)}}\right)$.
The analysis for the $Z^{\prime}$ is basically identical to that of the $V^{\prime}$ as far as concerns neutral currents. So we do not repeat it here. The unitarity bounds for both cases are collected in Table 4.

\subsection{Leptoquarks}

Let us start by first discussing the flavour structure of the leptoquark Lagrangian. Neglecting Lorentz and gauge indices, we have $\mathcal{L}_{L Q} \supset y_{Q L}^{i j} Q_{i} L_{j} \Phi+$ h.c., where $\Phi$ denotes one of the four leptoquarks in Table 3 . The simplest way to generate a contribution for either $R_{D^{(*)}}$ or $R_{K^{(*)}}$ is to switch on a single coupling, e.g. $y_{Q L}^{3 j}$, with the lepton index $j$ aligned either along the third or second generation. The $3 \rightarrow 2$ transition in the quark sector can then be obtained either via a $V_{c b}$ or $V_{t s}$ suppression. However, within such an approach the sign of the Wilson coefficient, which goes either like $\left|y_{Q L}^{3 j}\right|^{2} V_{c b}$ or $\left|y_{Q L}^{3 j}\right|^{2} V_{t s}$ (recall that $V_{c b}>0$ and $V_{t s}<0$ in the standard parametrization), is fixed and does not always correspond to the one necessary to reproduce the anomaly. ${ }^{7}$ Hence, in the following we define our simplified models based on the two leptoquark couplings $y_{Q L}^{3 j}$ and $y_{Q L}^{2 j}$, so that the sign of the contribution can be always matched. We further assume the scaling $y_{Q L}^{2 j} \sim y_{Q L}^{3 j} \lambda^{2}$, as suggested by motivated flavour structures. Given the hierarchy $y_{Q L}^{3 j} \gg y_{Q L}^{2 j}$, the scattering amplitudes are dominated by $y_{Q L}^{3 j}$ and the correlation of the partial wave in flavour space can be safely neglected. Indeed, for a leptoquark-mediated processes in the $t$-channel one should make the following replacement in the bound: $\left|y_{Q L}^{3 j}\right|^{2} \rightarrow \sqrt{\left|y_{Q L}^{3 j}\right|^{4}+\left|y_{Q L}^{2 j}\right|^{4}} \sim\left|y_{Q L}^{3 j}\right|^{2} \sqrt{1+\lambda^{4}}$, while no such flavour enhancement is even present for an $s$-channel scattering. Given these considerations, for each leptoquark of Table 2 we compute the unitarity constraints on its couplings and the matching condition with the effective operators in Eq. (1). Following the conventions of Ref. [44] we have:

- $S_{1} \sim(\overline{3}, 1,1 / 3): \mathcal{L}_{S_{1}} \supset y_{Q L}^{i j}{\overline{Q^{c}}}_{i, a} \epsilon^{a b} L_{j, b} S_{1}+$ h.c.

\footnotetext{
$\overline{7}$ With the single coupling $y_{Q L}^{3 j}$ we find that $S_{3}$ cannot explain neither of the anomalies, while $U_{3}$ cannot explain $R_{D^{(*)}}$.
} 
The strongest unitarity bound comes from the $t$-channel mediated $Q_{3}^{c}{\overline{Q^{c}}}_{3} \rightarrow L_{j} \bar{L}_{j}$ scattering, which in the limit $\sqrt{s} \gg M_{S_{1}}$ gives

$$
\left|y_{Q L}^{3 j}\right|^{2}<\frac{8 \pi}{\sqrt{3}}
$$

where we included a $\sqrt{3}$ enhancement factor due to the correlation of the partial wave in colour space. Integrating out $S_{1}$ and matching with the operators in Eq. (1) we obtain

$$
\frac{\left|y_{Q L}^{33}\right|^{2} \lambda^{2}}{2 M_{S_{1}}^{2}}=\frac{1}{\Lambda_{R_{D^{(*)}}}^{2}} .
$$

- $S_{3} \sim(\overline{3}, 3,1 / 3): \quad \mathcal{L}_{S_{3}} \supset y_{Q L}^{i j}{\overline{Q^{c}}}_{i, a}\left(\epsilon \sigma^{A}\right)^{a b} L_{j, b} S_{3}^{A}+$ h.c.

Analogously to the previous case we consider the $t$ channel mediated $Q_{3}^{c}{\overline{Q^{c}}}_{3} \rightarrow L_{j} \bar{L}_{j}$ scattering, from which we get the unitarity bound

$\left|y_{Q L}^{3 j}\right|^{2}<\frac{8 \pi}{3 \sqrt{3}}$,

where we included a $\sqrt{3} \times 3$ enhancement factor due to the correlation of the partial wave in the $S U(3)_{C} \times$ $S U(2)_{L}$ space. Integrating out $S_{3}$ and matching with the operators in Eq. (1) we obtain

$$
\frac{\left|y_{Q L}^{33}\right|^{2} \lambda^{2}}{2 M_{S_{3}}^{2}}=\frac{1}{\Lambda_{R_{D^{(*)}}}^{2}} \text { and } \quad \frac{\left|y_{Q L}^{32}\right|^{2} \lambda^{2}}{M_{S_{3}}^{2}}=\frac{1}{\Lambda_{R_{K^{(*)}}}^{2}} .
$$

- $U_{1} \sim(3,1,2 / 3): \mathcal{L}_{U_{1}} \supset y_{Q L}^{i j} \bar{Q}_{i, a} \gamma^{\mu} \delta^{a b} L_{j, b} U_{1, \mu}+$ h.c. By examining the $s$-channel process $\bar{Q}_{3} L_{j} \rightarrow \bar{Q}_{3} L_{j}$ at $\sqrt{s} \gg M_{U_{1}}$ we extract the unitarity bound ${ }^{8}$

$$
\left|y_{Q L}^{3 j}\right|^{2}<4 \pi
$$

where we included a factor 2 enhancement from the correlation of the partial wave in the $S U(2)_{L}$ space (while there is no $S U(3)_{C}$ enhancement since the colour flows through the diagram). Integrating out $U_{1}$ and matching with the operators in Eq. (1) we obtain

\footnotetext{
${ }^{8}$ The $t$-channel mediated $Q_{3} \bar{Q}_{3} \rightarrow L_{j} \bar{L}_{j}$ scattering cannot be straightforwardly used here, since the $J=0$ partial wave is formally divergent. This is due to the Coulomb singularity in the forward direction of the scattering for $\sqrt{s} \gg M_{U_{1}}$.
}

$$
\frac{\left|y_{Q L}^{33}\right|^{2} \lambda^{2}}{M_{U_{1}}^{2}}=\frac{1}{\Lambda_{R_{D^{(*)}}}^{2}} \text { and } \frac{\left|y_{Q L}^{32}\right|^{2} \lambda^{2}}{M_{U_{1}}^{2}}=\frac{1}{\Lambda_{R_{K^{*}}}^{2}} .
$$

- $U_{3} \sim(3,3,2 / 3): \mathcal{L}_{U_{3}} \supset y_{Q L}^{i j} \bar{Q}_{i, a} \gamma^{\mu}\left(\sigma^{A}\right)^{a b} L_{j, b} U_{3, \mu}^{A}+$ h.c.

Analogously to the previous case, from the $s$-channel process $\bar{Q}_{3} L_{j} \rightarrow \bar{Q}_{3} L_{j}$ we obtain

$\left|y_{Q L}^{3 j}\right|^{2}<4 \pi$.

Integrating out $U_{3}$ and matching with the operators in Eq. (1) we obtain

$$
\frac{\left|y_{Q L}^{33}\right|^{2} \lambda^{2}}{M_{U_{3}}^{2}}=\frac{1}{\Lambda_{R_{\left.D^{*}\right)}}^{2}} \text { and } \frac{\left|y_{Q L}^{32}\right|^{2} \lambda^{2}}{M_{U_{3}}^{2}}=\frac{1}{\Lambda_{R_{K^{(*)}}}^{2}} .
$$

After saturating the matching condition required to reproduce the anomalies, we can translate the unitarity bounds on the leptoquark couplings into an upper bound on the leptoquark masses. As a reference value we fix $\lambda^{2}=\left|V_{c b}\right|$ $\left(\left|V_{t s}\right|\right)$ for $R_{D^{(*)}}\left(R_{K^{(*)}}\right)$. The results are displayed in Table 5 , depending on the flavour structure of the leptoquark couplings.

\subsection{Direct searches at the LHC}

We will now briefly discuss the bounds from direct searches for the simplified models of Table 3 and compare them with the unitarity bounds on the new mediators' masses from Tables 4 and 5 . We will focus in particular on decay channels involving the third family, since these are theoretically motivated by flavour models and because it is precisely in those cases that the upper bounds on the mass of the new states are more stringent.

Let us discuss in turn the various cases. Reference [45] considered vector triplet $V^{\prime}$ exclusion limits by recasting $p p(b \bar{b}) \rightarrow \tau \bar{\tau}$ searches. The conclusion is that for relatively heavy vectors $M_{V^{\prime}} \gtrsim 500 \mathrm{GeV}$ the resolution of the $R_{D^{(*)}}$ anomaly with dominant third generation couplings requires a very large $Z^{\prime}$ decay width (where here $Z^{\prime}$ denotes here the neutral component of $V^{\prime}$ ), which is beyond the perturbative regime. This is somehow compatible with our unitarity bound $M_{V^{\prime}}<1.3 \mathrm{TeV}$ in Table 4. Note, however, that a large $Z^{\prime}$ width also implies extra model-dependent decay channels which would in principle contribute to our scattering amplitudes, and would yield in turn a bound stronger than 1.3 $\mathrm{TeV}$. On the other hand, for light masses $M_{Z^{\prime}} \lesssim 400 \mathrm{GeV}$ a perturbative window with a relatively small $Z^{\prime}$ width is 
Table 5 Summary of unitarity bounds for leptoquarks. $\mathrm{FS}_{Q}$ and $\mathrm{FS}_{L}$ indicate the flavour suppression factors in the quark and lepton sectors (same as in Table 1). Bold (italics) values denote the cases excluded (disfavoured) by direct searches (see Sect. 6.3)

\begin{tabular}{llllllll}
\hline Anomaly & Coupling & $\mathrm{FS}_{Q}$ & $\mathrm{FS}_{L}$ & $M_{S_{1}}(\mathrm{TeV})$ & $M_{S_{3}}(\mathrm{TeV})$ & $M_{U_{1}}(\mathrm{TeV})$ & $M_{U_{3}}(\mathrm{TeV})$ \\
\hline$b \rightarrow c \tau \bar{v}$ & $y_{Q L}^{33}$ & $\left|V_{c b}\right|$ & 1 & 1.3 & $\mathbf{0 . 8}$ & 1.7 & 1.7 \\
$b \rightarrow s \mu \bar{\mu}$ & $y_{Q L}^{32}$ & $\left|V_{t s}\right|$ & 1 & $\times$ & 3.3 & 22 & 5.4 \\
$b \rightarrow s \mu \bar{\mu}$ & $y_{Q L}^{33}$ & $\left|V_{t s}\right|$ & $m_{\mu} / m_{\tau}$ & $\times$ & $\mathbf{0 . 8}$ & 1.3 & 1.3 \\
$b \rightarrow s \mu \bar{\mu}$ & $y_{Q L}^{33}$ & $\left|V_{t s}\right|$ & $\left(m_{\mu} / m_{\tau}\right)^{2}$ & $\times$ & & 1.3 \\
\hline
\end{tabular}

not yet excluded by $\tau \bar{\tau}$ searches. However, this requires a suppression of electroweak precision observables which are generically quite constraining [46].

If leptoquarks are light enough they can be pair-produced at LHC with sizeable cross-section via QCD interactions. As already stated we assume that decay channels are dominated by third generation SM fermions. $S_{1}$ has the same quantum numbers of a sbottom and decays into either $S_{1} \rightarrow \bar{b} \bar{v}_{\tau}$ or $S_{1} \rightarrow \bar{t} \bar{\tau}$ (both with $\mathcal{B}=50 \%$ ). Using the results of [47] we obtain $M_{S_{1}}>570 \mathrm{GeV}$, which is still compatible with the unitarity bound in Table 5 . On the other hand, $S_{3}$ comprises three charge eigenstates, respectively with charges $4 / 3,1 / 3$ and $-2 / 3$. The predominant decays are $S_{3}^{4 / 3} \rightarrow \bar{b} \bar{\tau}$, $S_{3}^{1 / 3} \rightarrow \bar{b} \bar{v}_{\tau}$ or $S_{3}^{1 / 3} \rightarrow \bar{t} \bar{\tau}$ (both with $\mathcal{B}=50 \%$ ) and $S_{3}^{-2 / 3} \rightarrow \bar{t} \bar{\nu}_{\tau}$. There will be electroweak mass splittings between the three leptoquark states, allowing the heavier ones to decay to the lighter ones, but these decays will be subdominant to those through the leptoquark couplings, if the mass splittings are small. In fact, by using the results of Ref. [48] we find that electroweak precision data exclude mass splittings within $S_{3}$ above $\mathcal{O}(25) \mathrm{GeV}$. For $S_{3}^{-2 / 3}$ we can infer a bound of $M_{S_{3}^{-2 / 3}} \gtrsim 950 \mathrm{GeV}$, by looking at SUSY searches for $\tilde{t} \rightarrow t \tilde{\chi}_{0}$ [49]. For $S_{3}^{4 / 3}$ there is a dedicated leptoquark search for third generation final states [50], which yields $S_{3}^{4 / 3} \gtrsim 850 \mathrm{GeV}$. The bound on $S_{3}^{1 / 3}$ basically corresponds to the previous one for $S_{1}$. All in all, when comparing the limits from direct searches with the unitarity bounds in Table 5, we conclude that a leptoquark $S_{3}$ with couplings dominantly aligned along the third generation cannot explain within a perturbative framework the $R_{D^{(*)}}$ anomaly (and $R_{K^{(*)}}$ as well, under the hypothesis of MFV in the lepton sector).

We finally discuss vector leptoquarks. Under the assumption of leading third generation couplings one can look at $p p(b \bar{b}) \rightarrow \tau \bar{\tau}$ searches, which however are not yet sensitive enough to rule out the explanation of $R_{D^{(*)}}$ via $U_{1}$ [45]. On the other hand, vector leptoquarks can also be efficiently pair-produced at LHC via their coupling to gluons. This interaction depends however on the UV completion of the vector. The most general CP-conserving Lagrangian describing the interaction of the vector $U_{\mu}$ with gluons (including operators up to $d=4$ ) is given by [51]

$$
\begin{aligned}
\mathcal{L}_{U}^{g}= & -\frac{1}{2}\left(D_{[\mu} U_{\nu]}\right)^{\dagger} D^{[\mu} U^{\nu]}+M_{U}^{2} U_{\mu}^{\dagger} U^{\mu} \\
& -i g_{s}\left(1-\kappa_{G}\right) U_{\mu}^{\dagger} t^{a} U_{\nu} G^{a \mu \nu},
\end{aligned}
$$

where $D_{\mu}=\partial_{\mu}-i g_{s} t^{a} G_{\mu}^{a}$ is the QCD covariant derivative and $G_{\mu \nu}^{a}=\partial_{\mu} G_{\nu}^{a}-\partial_{\nu} G_{\mu}^{a}+g_{s} f^{a b c} G_{\mu}^{b} G_{\nu}^{c}$ is the usual QCD field strength. As two benchmark scenarios we consider the minimal coupling (MC) and the Yang Mills (YM) type of coupling of Ref. [51]. The former case $\left(\kappa_{G}=1\right)$, refers to the interaction stemming purely from the $\mathrm{QCD}$ covariant derivative of the vector, while the latter $\left(\kappa_{G}=0\right)$ includes non-minimal interactions between the vector and the gluons which arise when the vector has a gauge origin. ${ }^{9}$ We remark, however, that $\kappa_{G}$ is an unknown parameter.

In the exact $U(2)$ flavour limit $U_{1}$ decays in either $U_{1} \rightarrow t \bar{v}_{\tau}$ or $U_{1} \rightarrow b \bar{\tau}$ (both with $\mathcal{B}=50 \%$ ). By revisiting a $\sqrt{s}=8 \mathrm{TeV}$ ATLAS search [53] for QCD pairproduced third generation scalar leptoquark in the $t \bar{t} v \bar{v}$ channel, Ref. [38] excludes $M_{U_{1}}<770 \mathrm{GeV}$. The latter exclusion actually applies to the MC scenario. In the meanwhile, there has been a new analysis at $\sqrt{s}=13 \mathrm{TeV}$ [50] for searches of scalar leptoquarks decaying in third generation SM fermions. We perform a rescaling of the bounds by employing the results in Ref. [51] on the vector leptoquark total cross-section and extract the bounds: $M_{U_{1}} \gtrsim 1.0 \mathrm{TeV}$ (MC case) and $M_{U_{1}} \gtrsim 1.3 \mathrm{TeV}$ (YM case). Given the vicinity to the unitarity bounds in Table 5, we remark that a dedicated experimental search in this case would be very helpful. Finally, since $U_{3}$ contains an isospin component with the same charge of $U_{1}$ we expect similar bounds, though optimized searches for the other charge eigenstates might yield better constraints.

\section{Conclusions}

In this work we have investigated the constraints of partialwave unitarity for the new physics interpretation of the recent hints of LFU violation in $B$-meson semi-leptonic decays,

\footnotetext{
9 The explanation of $R_{D^{(*)}}$ and/or $R_{K^{(*)}}$ via gauge leptoquarks is strongly disfavoured. In fact, gauge invariance enforces extra constraints on the vector Lagrangian, like e.g. the unitarity of the leptoquark interactions in flavour space (see [52] for a recent discussion).
} 
both within an EFT approach and by employing simplified models. In order to simplify the discussion we focussed on a single $S U(2)_{L}$ triplet operator (cf. Eq. (4)) which can contribute to both $R_{D^{(*)}}$ or $R_{K^{(*)}}$, but without necessarily relying on the common explanation of both anomalies. This can be straightforwardly extended to the more general situation involving multiple operators, by employing the results of Sect. 5 in which we derived the connection between the scale of unitarity violation $\Lambda_{U}$ and the coefficients $\Lambda_{\mathcal{O}}$ of the semi-leptonic SMEFT operator basis.

The results of the EFT analysis are summarized in Table 1. In particular, we find that the most conservative bound on the scale of unitarity violation is $\Lambda_{U}=9.2$ and 84 $\mathrm{TeV}$, respectively for $R_{D^{(*)}}$ or $R_{K^{(*)}}$. This corresponds to the case when the effective operators are aligned in the direction of the flavour eigenstates responsible for the anomalies. On the other hand, motivated frameworks like e.g. MFV, $U$ (2) flavour models and PC suggest an alignment of the effective operators along the third generation, thus implying that stronger unitarity bounds can be actually extracted by considering third generation fermions' scatterings. For instance, in the case of third generation alignment in the quark sector the previous bounds become $\Lambda_{U}=1.9$ and $17 \mathrm{TeV}$, with the latter reaching even few $\mathrm{TeV}$ in the case of hierarchical flavour structures also in the lepton sector.

In a similar way one can use the tool of perturbative unitarity to set constraints on the parameter space of simplified models explaining the $B$-flavour anomalies. As a representative class of models we considered colourless vectors and scalar/vector leptoquarks (cf. Table 3). In all those cases, it was possible to use partial-wave unitarity in order to set bounds on the renormalizable couplings of the new mediators with the SM fermions. By fixing the ratio coupling/mass in order to fit the anomaly, the unitarity bound was hence translated into an upper bound on the mass of the simplified model's mediator. The results are collected in Tables 4 and 5 for some reference flavour structures.

While for the anomalies in $b \rightarrow s \ell \bar{\ell}$ transitions it is much easier to accommodate direct searches, that is not the case for $R_{D^{(*)}}$. Simplified models for explaining the latter are problematic for various reasons: $d_{i} \rightarrow d_{j} v \bar{v}$ transitions, electroweak precision observables, radiative generation of LFU breaking effects in $Z$ and $\tau$ decays, etc. On top of that, one should take into account unitarity constraints within perturbatively calculable models. The vector leptoquark $U_{1}$ seems phenomenologically in a better shape for explaining $R_{D^{(*)}}$, since it is automatically free from issues like $d_{i} \rightarrow d_{j} \nu \bar{v}$ transitions and also because, being an $S U(2)_{L}$ singlet, bounds from electroweak precision data are more easily evaded. For this specific case we provided a new bound by rescaling recent searches at LHC Run-2 with full dataset, finding that $M_{U_{1}} \gtrsim 1 \div 1.3 \mathrm{TeV}$ (depending on the UV comple- tion of the vector). In those cases where the leptoquark couplings are dominantly aligned along the third generation, the open window between direct searches and perturbativity is quite reduced and might be eventually closed in the near future.

Acknowledgements We thank Dario Buttazzo, Roberto Franceschini, Ramona Gröber, Jorge Martin Camalich, David Marzocca, Enrico Nardi, Giovanni Ridolfi, Alessandro Strumia and Riccardo Torre for helpful discussions, Jernej F. Kamenik for collaboration in the early stages of this project and Gian Francesco Giudice for encouraging support.

Open Access This article is distributed under the terms of the Creative Commons Attribution 4.0 International License (http://creativecomm ons.org/licenses/by/4.0/), which permits unrestricted use, distribution, and reproduction in any medium, provided you give appropriate credit to the original author(s) and the source, provide a link to the Creative Commons license, and indicate if changes were made.

Funded by SCOAP . $^{3}$.

\section{A Sample calculation: $S U(2)_{L}$ triplet operator}

In this appendix we exemplify the calculation of the unitarity bound in the presence of the triplet operator

$\frac{1}{\Lambda_{Q L^{(3)}}^{2}}\left(\bar{Q}_{L} \gamma^{\mu} \sigma^{A} Q_{L}\right)\left(\bar{L}_{L} \gamma_{\mu} \sigma^{A} L_{L}\right)$

We are interested in evaluating the scattering amplitude

$Q(p, r, a, \alpha)+\bar{Q}(k, s, b, \beta) \rightarrow L\left(p^{\prime}, r^{\prime}, c\right)+\bar{L}\left(k^{\prime}, s^{\prime}, d\right)$

where the indices $(p, r, a, \alpha)$ denote respectively momentum, polarization, $S U(2)_{L}$ and colour indices. The Lorentz invariant matrix element is given by

$$
\begin{aligned}
\mathcal{M}= & -\frac{1}{4 \Lambda_{Q L^{(3)}}^{2}} \delta_{\alpha \beta}\left(\sigma^{A}\right)_{a b}\left(\sigma^{A}\right)_{c d} \\
& \times\left(\bar{v}^{s}(k) \gamma_{\mu}\left(1-\gamma_{5}\right) u^{r}(p) \bar{u}^{r^{\prime}}\left(p^{\prime}\right) \gamma^{\mu}\left(1-\gamma_{5}\right) v^{s^{\prime}}\left(k^{\prime}\right)\right) .
\end{aligned}
$$

Since in the massless limit the fermions in Eq. (39) are helicity eigenstates, at energies $\sqrt{s} \gg v$ only the +--+ polarization survives, yielding ${ }^{10}$

$\mathcal{M}_{+--+}(\sqrt{s}, \cos \theta) \stackrel{\sqrt{s} \gg v}{\simeq} \frac{2}{\Lambda_{Q L^{(3)}}^{2}} \delta_{\alpha \beta}\left(\sigma^{A}\right)_{a b}\left(\sigma^{A}\right)_{c d} s \cos ^{2} \frac{\theta}{2}$

10 We refer to Appendix A.2 of Ref. [33] for the explicit representation of the spinorial variables. 
The $J=0$ partial-wave scattering matrix is obtained via

$$
\begin{aligned}
a^{0} & \stackrel{\sqrt{s} \gg v}{\simeq} \frac{1}{32} \int_{-1}^{+1} \mathrm{~d}(\cos \theta) \mathcal{M}_{+--+}(\sqrt{s}, \cos \theta) \\
& =\frac{s}{16 \pi} \frac{1}{\Lambda_{Q L^{(3)}}^{2}} \delta_{\alpha \beta}\left(\sigma^{A}\right)_{a b}\left(\sigma^{A}\right)_{c d} .
\end{aligned}
$$

In order to maximize the unitarity bound one can prepare the scattering eigenstates in such a way that they correspond to the highest eigenvalues of $a^{0}$ in the gauge group space. Let us discuss in turn the $S U(3)_{C}$ and $S U(2)_{L}$ structures. In the former case the partial wave can be represented via the matrix (defined on the basis $\left.\left\{\left(Q_{L}\right)^{1}\left(\bar{Q}_{L}\right)^{1},\left(Q_{L}\right)^{2}\left(\bar{Q}_{L}\right)^{2},\left(Q_{L}\right)^{3}\left(\bar{Q}_{L}\right)^{3},\left(L_{L}\right)\left(\bar{L}_{L}\right)\right\}\right)$

$a_{S U(3)_{C}}^{0}=\left(\begin{array}{cccc}0 & 0 & 0 & 1 \\ 0 & 0 & 0 & 1 \\ 0 & 0 & 0 & 1 \\ 1 & 1 & 1 & 0\end{array}\right)$,

whose eigenvalues are $(\sqrt{3},-\sqrt{3}, 0,0)$. Thus, by preparing the initial and final states of the scattering in the eigenstate $\frac{1}{\sqrt{6}}(1,1,1, \sqrt{3})$, the colour enhancement factor corresponds to $\sqrt{3}$. On the other hand, the partial wave in the $S U(2)_{L}$ space has the matrix form

$a_{S U(2)_{L}}^{0}=\left(\begin{array}{cccc}1 & 0 & 0 & -1 \\ 0 & 0 & 2 & 0 \\ 0 & 2 & 0 & 0 \\ -1 & 0 & 0 & 1\end{array}\right)$,

defined on the basis $\left\{\psi_{1} \bar{\psi}_{1}, \psi_{1} \bar{\psi}_{2}, \psi_{2} \bar{\psi}_{1}, \psi_{2} \bar{\psi}_{2}\right\}$, where $\psi_{a}$ ( $a=1,2$ being an $S U(2)_{L}$ index) denotes either $\left(Q_{L}\right)^{\alpha}$ or $L_{L}$. In order to derive Eq. (45) it is convenient to use the Fierz identity $\left(\sigma^{A}\right)_{a b}\left(\sigma^{A}\right)_{c d}=2 \delta_{a d} \delta_{c b}-\delta_{a b} \delta_{c d}$. Since the eigenvalues of $a_{S U(2)_{L}}^{0}$ are $(2,2,-2,0)$, by preparing the initial and final states of the scattering in the eigenstate $\frac{1}{\sqrt{2}}(0,1,1,0)$, the $S U(2)_{L}$ enhancement factor is 2 . Summarizing, the gauge group enhancement leads to an extra $\sqrt{3} \times 2$ factor in the partial-wave eigenvalue, and including the latter we obtain

$a^{0}=\frac{\sqrt{3}}{8 \pi} \frac{s}{\Lambda_{Q L^{(3)}}^{2}}$.

From the condition in Eq. (19) it finally the unitarity bound $\sqrt{s}<\Lambda_{U}$ follows, where

$\Lambda_{U}=\sqrt{\frac{4 \pi}{\sqrt{3}}}\left|\Lambda_{Q L^{(3)}}\right|$

As a final remark, we briefly mention an alternative way to work out the gauge group enhancement which employs irreducible representations for the scattering amplitude [33]. Denoting by $\psi_{i}\left(\bar{\psi}_{j}\right)$ the fundamental (anti-fundamental) representation of an $S U(N)$ group, a general two-particle state $\left|\psi_{i} \bar{\psi}_{j}\right\rangle$ can be decomposed into a singlet and an adjoint channel,

$$
\begin{aligned}
& |\psi \bar{\psi}\rangle_{\mathbf{1}}=\frac{\delta_{i j}}{\sqrt{N}}\left|\psi_{i} \bar{\psi}_{j}\right\rangle, \\
& |\psi \bar{\psi}\rangle_{\text {Adj }}^{A}=T_{i j}^{A}\left|\psi_{i} \bar{\psi}_{j}\right\rangle,
\end{aligned}
$$

where $T^{A}$, with $A=1, \ldots, N^{2}-1$, are $S U(N)$ generators (in the normalization $\operatorname{Tr} T^{A} T^{B}=\delta^{A B}$ ) and we properly normalized the states to unitary norm. The scattering amplitude in Eq. (42) has both $S U(3)_{C}$ and $S U(2)_{L}$ components. In the former case the $\mathcal{S}$-matrix elements in the (colour) singlet and adjoint channels are

$$
\begin{aligned}
\langle L \bar{L}|\mathcal{S}| Q \bar{Q}\rangle_{\mathbf{1}} & =\frac{\delta_{\alpha \beta}}{\sqrt{3}}\left\langle L \bar{L}|\mathcal{S}| Q_{\alpha} \bar{Q}_{b}\right\rangle \\
& =\frac{\delta_{\alpha \beta}}{\sqrt{3}} \mathcal{M}_{S U(3)_{C}} \delta_{\alpha \beta}=\sqrt{3} \mathcal{M}_{S U(3)_{C}}, \\
\langle L \bar{L}|\mathcal{S}| Q \bar{Q}\rangle_{\text {Adj }}^{A} & =T_{\alpha \beta}^{A}\left\langle L \bar{L}|\mathcal{S}| Q_{\alpha} \bar{Q}_{\beta}\right\rangle \\
& =T_{\alpha \beta}^{A} \mathcal{M}_{S U(3)_{C}} \delta_{\alpha \beta}=0,
\end{aligned}
$$

where $\mathcal{M}_{S U(3)_{C}}$ denotes the matrix element in Eq. (42) stripped from the colour structure. For the $S U(2)_{L}$ case istead let us collectively denote the doublets (either $Q$ or $L$ ) by $\psi_{a}$, with $a=1,2$ being an $S U(2)_{L}$ index. Then the singlet and adjoint scattering channels are

$$
\begin{aligned}
1\langle\psi \bar{\psi}|\mathcal{S}| \psi \bar{\psi}\rangle_{\mathbf{1}} & =\frac{\delta_{a b} \delta_{c d}}{2}\left\langle\psi_{a} \bar{\psi}_{b}|\mathcal{S}| \psi_{c} \bar{\psi}_{d}\right\rangle \\
& =\frac{\delta_{a b} \delta_{c d}}{2} \mathcal{M}_{S U(2)_{L}}\left(2 \delta_{a d} \delta_{c b}-\delta_{a b} \delta_{c d}\right) \\
& =\frac{1}{2} \mathcal{M}_{S U(2)_{L}}\left(2 \delta_{a a}-\delta_{a a} \delta_{c c}\right)=0 \\
{ }_{\text {Adj }}^{A}\langle\psi \bar{\psi}|\mathcal{S}| \psi \bar{\psi}\rangle_{\text {Adj }}^{B} & =T_{a b}^{A} T_{c d}^{B}\left\langle\psi_{a} \bar{\psi}_{b}|\mathcal{S}| \psi_{c} \bar{\psi}_{d}\right\rangle \\
& =T_{a b}^{A} T_{c d}^{B} \mathcal{M}_{S U(2)_{L}}\left(2 \delta_{a d} \delta_{c b}-\delta_{a b} \delta_{c d}\right) \\
& =\mathcal{M}_{S U(2)_{L}}\left(2 \operatorname{Tr}\left(T^{A} T^{B}\right)-\operatorname{Tr}\left(T^{A}\right) \operatorname{Tr}\left(T^{B}\right)\right) \\
& =2 \delta^{A B} \mathcal{M}_{S U(2)_{L}},
\end{aligned}
$$

where $\mathcal{M}_{S U(2)_{C}}$ denotes the matrix element in Eq. (42) stripped from the $S U(2)_{L}$ structure. Hence, by considering the singlet channel in colour space and the adjoint channel in $S U(2)_{L}$ space, we gain respectively a factor $\sqrt{3}$ and 2 in the partial wave.

\section{References}

1. LHCb Collaboration, R. Aaij et al., Differential branching fractions and isospin asymmetries of $B \rightarrow K^{(*)} \mu^{+} \mu^{-}$decays. JHEP06, 133 (2014). arXiv:1403.8044 [hep-ex] 
2. LHCb Collaboration, R. Aaij et al., Measurement of form-factorindependent observables in the decay $B^{0} \rightarrow K^{* 0} \mu^{+} \mu^{-}$. Phys. Rev. Lett.111, 191801 (2013). arXiv:1308.1707 [hep-ex]

3. LHCb Collaboration, R. Aaij et al., Angular analysis of the $B^{0} \rightarrow$ $K^{* 0} \mu^{+} \mu^{-}$decay using $3 \mathrm{fb}^{-1}$ of integrated luminosity. JHEP02, 104 (2016). arXiv:1512.04442 [hep-ex]

4. LHCb Collaboration, R. Aaij et al., Angular analysis and differential branching fraction of the decay $B_{s}^{0} \rightarrow \phi \mu^{+} \mu^{-}$. JHEP 09, 179 (2015). arXiv:1506.08777 [hep-ex]

5. LHCb Collaboration, R. Aaij et al., Test of lepton universality using $B^{+} \rightarrow K^{+} \ell^{+} \ell^{-}$decays. Phys. Rev. Lett. 113, 151601 (2014). arXiv:1406.6482 [hep-ex]

6. LHCb Collaboration, R. Aaij et al., Test of lepton universality with $B^{0} \rightarrow K^{* 0} \ell^{+} \ell^{-}$decays. arXiv: 1705.05802 [hep-ex]

7. M. Bordone, G. Isidori, A. Pattori, On the Standard Model predictions for $R_{K}$ and $R_{K^{*}}$. Eur. Phys. J. C 76(8), 440 (2016). arXiv:1605.07633 [hep-ph]

8. B. Capdevila, A. Crivellin, S. Descotes-Genon, J. Matias, J. Virto, Patterns of new physics in $b \rightarrow s \ell^{+} \ell^{-}$transitions in the light of recent data. arXiv:1704.05340 [hep-ph]

9. W. Altmannshofer, P. Stangl, D.M. Straub, Interpreting hints for lepton flavor universality violation. arXiv:1704.05435 [hep-ph]

10. G. D’Amico, M. Nardecchia, P. Panci, F. Sannino, A. Strumia, R. Torre, A. Urbano, Flavour anomalies after the $R_{K^{*}}$ measurement. arXiv:1704.05438 [hep-ph]

11. M. Ciuchini, A.M. Coutinho, M. Fedele, E. Franco, A. Paul, L. Silvestrini, M. Valli, On flavourful Easter eggs for new physics hunger and lepton flavour universality violation. arXiv:1704.05447 [hep$\mathrm{ph}]$

12. L.-S. Geng, B. Grinstein, S. Jager, J. Martin Camalich, X.-L. Ren, R.-X. Shi, Towards the discovery of new physics with leptonuniversality ratios of $b \rightarrow$ sll decays. arXiv:1704.05446 [hep-ph]

13. BaBar Collaboration, J.P. Lees et al., Measurement of an excess of $\bar{B} \rightarrow D^{(*)} \tau^{-} \bar{v}_{\tau}$ decays and implications for charged Higgs bosons. Phys. Rev. D 88(7), 072012 (2013). arXiv:1303.0571 [hep-ex]

14. LHCb Collaboration, R. Aaij et al., Measurement of the ratio of branching fractions $\mathcal{B}\left(\bar{B}^{0} \rightarrow D^{*+} \tau^{-} \bar{v}_{\tau}\right) / \mathcal{B}\left(\bar{B}^{0} \rightarrow D^{*+} \mu^{-} \bar{v}_{\mu}\right)$. Phys. Rev. Lett. 115(11), 111803 (2015). arXiv:1506.08614 [hepex]. [Erratum: Phys. Rev. Lett. 115, no. 15, 159901 (2015)]

15. S. Belle Collaboration, Hirose et al., "Measurement of the $\tau$ lepton polarization and $R\left(D^{*}\right)$ in the decay $\bar{B} \rightarrow D^{*} \tau^{-} \bar{\nu}_{\tau}$, ,. Phys. Rev. Lett. 118(21), 211801 (2017). arXiv:1612.00529 [hep-ex]

16. F.U. Bernlochner, Z. Ligeti, M. Papucci, D.J. Robinson, Combined analysis of semileptonic $B$ decays to $D$ and $D^{*}: R\left(D^{(*)}\right),\left|V_{c b}\right|$, and new physics. arXiv:1703.05330 [hep-ph]

17. LHCb Collaboration, Lepton flavour universality tests using semitauonic decays at LHCb. Antonio Romero Vidal, LHC Seminar presented at CERN, 06/06/2017

18. B.W. Lee, C. Quigg, H.B. Thacker, The strength of weak interactions at very high-energies and the Higgs boson mass. Phys. Rev. Lett. 38, 883-885 (1977)

19. B.W. Lee, C. Quigg, H.B. Thacker, Weak interactions at very highenergies: the role of the Higgs boson mass. Phys. Rev. D 16, 1519 (1977)

20. E. Fermi, Tentativo di una Teoria Dei Raggi $\beta$. Nuovo Cim. 11, 1-19 (1934)

21. C.M. Becchi, G. Ridolfi, An Introduction to Relativistic Processes and the Standard Model of Electroweak Interactions (Springer, Berlin, 2014)

22. U. Aydemir, M.M. Anber, J.F. Donoghue, Self-healing of unitarity in effective field theories and the onset of new physics. Phys. Rev. D 86, 014025 (2012). arXiv:1203.5153 [hep-ph]

23. R. Alonso, B. Grinstein, J. Martin Camalich, Lepton universality violation and lepton flavor conservation in $B$-meson decays. JHEP 10, 184 (2015). arXiv:1505.05164 [hep-ph]
24. A. Manohar, H. Georgi, Chiral quarks and the nonrelativistic quark model. Nucl. Phys. B 234, 189-212 (1984)

25. A.G. Cohen, D.B. Kaplan, A.E. Nelson, Counting 4 pis in strongly coupled supersymmetry. Phys. Lett. B 412, 301-308 (1997). arXiv:hep-ph/9706275 [hep-ph]

26. G. Panico, A. Wulzer, The composite Nambu-Goldstone Higgs. Lect. Notes Phys. 913, 1-316 (2016). arXiv:1506.01961 [hep-ph]

27. G. D'Ambrosio, G.F. Giudice, G. Isidori, A. Strumia, Minimal flavor violation: an effective field theory approach. Nucl. Phys. B 645, 155-187 (2002). arXiv:hep-ph/0207036 [hep-ph]

28. R. Barbieri, G. Isidori, J. Jones-Perez, P. Lodone, D.M. Straub, $U(2)$ and minimal flavour violation in supersymmetry. Eur. Phys. J. C 71, 1725 (2011). arXiv:1105.2296 [hep-ph]

29. D.B. Kaplan, Flavor at SSC energies: a new mechanism for dynamically generated fermion masses. Nucl. Phys. B 365, 259-278 (1991)

30. B. Keren-Zur, P. Lodone, M. Nardecchia, D. Pappadopulo, R. Rattazzi, L. Vecchi, On partial compositeness and the CP asymmetry in charm decays. Nucl. Phys. B 867, 394-428 (2013). arXiv:1205.5803 [hep-ph]

31. C. Itzykson, J.B. Zuber, Quantum Field Theory. International Series in Pure and Applied Physics (McGraw-Hill, New York, 1980). doi:10.1063/1.2916419

32. M.S. Chanowitz, M.A. Furman, I. Hinchliffe, Weak interactions of ultraheavy fermions. 2. Nucl. Phys. B 153, 402 (1979)

33. L. Di Luzio, J.F. Kamenik, M. Nardecchia, Implications of perturbative unitarity for scalar di-boson resonance searches at LHC. Eur. Phys. J. C 77(1), 30 (2017). arXiv:1604.05746 [hep-ph]

34. A.J. Buras, J. Girrbach-Noe, C. Niehoff, D.M. Straub, $B \rightarrow$ $K^{(*)} \nu \bar{v}$ decays in the Standard Model and beyond. JHEP 02, 184 (2015). arXiv:1409.4557 [hep-ph]

35. M. Bordone, D. Buttazzo, G. Isidori, J. Monnard, Probing lepton flavour universality with $K \rightarrow \pi \nu \bar{v}$ decays. arXiv:1705.10729 [hep-ph]

36. A. Crivellin, D. Muller, T. Ota, Simultaneous explanation of $R\left(D^{(*)}\right)$ and $b \rightarrow s \mu^{+} \mu^{-}$: the last scalar leptoquarks standing. arXiv:1703.09226 [hep-ph]

37. L. Calibbi, A. Crivellin, T. Ota, Effective field theory approach to $b \rightarrow \operatorname{se\ell }^{\left({ }^{\prime}\right)}, B \rightarrow K^{(*)} \nu \bar{v}$ and $B \rightarrow D^{(*)} \tau \nu$ with third generation couplings. Phys. Rev. Lett. 115, 181801 (2015). arXiv:1506.02661 [hep-ph]

38. R. Barbieri, G. Isidori, A. Pattori, F. Senia, Anomalies in $B$-decays and $U(2)$ flavour symmetry. Eur. Phys. J. C 76(2), 67 (2016). arXiv: 1512.01560 [hep-ph]

39. R. Barbieri, C.W. Murphy, F. Senia, B-decay anomalies in a composite leptoquark model. Eur. Phys. J. C 77(1), 8 (2017). arXiv:1611.04930 [hep-ph]

40. F. Feruglio, P. Paradisi, A. Pattori, Revisiting lepton flavor universality in B decays. Phys. Rev. Lett. 118(1), 011801 (2017). arXiv:1606.00524 [hep-ph]

41. F. Feruglio, P. Paradisi, A. Pattori, On the importance of electroweak corrections for B anomalies. arXiv:1705.00929 [hep-ph]

42. W. Altmannshofer, P.S.B. Dev, A. Soni, $R_{D^{(*)}}$ anomaly: a possible hint for natural supersymmetry with $R$-parity violation. arXiv:1704.06659 [hep-ph]

43. A. Greljo, G. Isidori, D. Marzocca, On the breaking of lepton flavor universality in B decays. JHEP 07, 142 (2015). arXiv:1506.01705 [hep-ph]

44. I. Dorsner, S. Fajfer, A. Greljo, J.F. Kamenik, N. Kosnik, Physics of leptoquarks in precision experiments and at particle colliders. Phys. Rep. 641, 1-68 (2016). arXiv:1603.04993 [hep-ph]

45. D.A. Faroughy, A. Greljo, J.F. Kamenik, Confronting lepton flavor universality violation in B decays with high- $p_{T}$ tau lepton searches at LHC. Phys. Lett. B 764, 126-134 (2017). arXiv:1609.07138 [hep-ph] 
46. D. Pappadopulo, A. Thamm, R. Torre, A. Wulzer, Heavy vector triplets: bridging theory and data. JHEP 09, 060 (2014). arXiv:1402.4431 [hep-ph]

47. B. Gripaios, M. Nardecchia, S.A. Renner, Composite leptoquarks and anomalies in $B$-meson decays. JHEP 05, 006 (2015). arXiv:1412.1791 [hep-ph]

48. L. Di Luzio, R. Grober, J.F. Kamenik, M. Nardecchia, Accidental matter at the LHC. JHEP 07, 074 (2015). arXiv:1504.00359 [hep$\mathrm{ph}]$

49. ATLAS Collaboration, Search for a scalar partner of the top quark in the Jets + ETmiss final state at $\sqrt{s}=13 \mathrm{TeV}$ with the ATLAS detector. ATLAS-CONF-2017-020

50. CMS Collaboration, A.M. Sirunyan et al., Search for the thirdgeneration scalar leptoquarks and heavy right-handed neutrinos in final states with two tau leptons and two jets in proton-proton collisions at $\sqrt{s}=13 \mathrm{TeV}$. arXiv:1703.03995 [hep-ex]. CMS-EXO16-023, CERN-EP-2017-025
51. J. Blumlein, E. Boos, A. Kryukov, Leptoquark pair production in hadronic interactions. Z. Phys. C 76, 137-153 (1997). arXiv:hep-ph/9610408 [hep-ph]

52. C. Biggio, M. Bordone, L. Di Luzio, G. Ridolfi, Massive vectors and loop observables: the $g-2$ case. JHEP 10, 002 (2016). arXiv:1607.07621 [hep-ph]

53. ATLAS Collaboration, G. Aad et al., Searches for scalar leptoquarks in pp collisions at $\sqrt{s}=8 \mathrm{TeV}$ with the ATLAS detector. Eur. Phys. J. C 76(1), 5 (2016). arXiv:1508.04735 [hep-ex] 